\title{
Fentanyl-Induced Chest Wall Rigidity as a Cause of Acute Respiratory Failure in the Intensive Care Unit
}

\author{
Peh Wee Minga, c, Darshan Lalit Singh ${ }^{\mathrm{b}}$
}

\begin{abstract}
We aim to report a case of chest wall rigidity induced by high-dose fentanyl infusion sedation and analgesia in the intensive care unit (ICU) for management of pneumonia and asthma. The patient is an 80-year-old woman, who presented to the hospital with complaints of fever and productive cough with yellowish expectoration of 2 days duration. She also had lethargy over the same time period and had sick contacts in the form of two daughters who both had recently recovered from a "flu-like" illness. She was known to have bronchial asthma treated with seretide 25/250 two puffs ON + PO monteleukast $10 \mathrm{mg} \mathrm{ON}$, hypertension treated with PO losartan $50 \mathrm{mg} \mathrm{BD}$, type 2 diabetes mellitus controlled with PO linagliptin $5 \mathrm{mg}$ OM and a previous right thalamic ischemic stroke 5 years ago for which she was on PO clopidogrel $75 \mathrm{mg} \mathrm{OM}$ and PO simvastatin. She developed severe ventilator desynchrony characterized by dramatic sudden onset of severe hypercarbia, severely decreased pulmonary compliance and episodic breath holding. She was empirically treated for asthma exacerbation and treated with steroids, bronchodilators and manual ventilation but despite doing so during this episode the patient failed to respond. The patient was clinically evaluated and dynamic hyperinflation was excluded as a cause of the respiratory failure. There was no evidence of pneumothorax and worsening pneumonia. Considerations of insufficient sedation and analgesia led to deepening sedation and analgesia without good response. Ventilation was dramatically improved after small doses of neuromuscular relaxation. This cycle was repeated many times. The patient was kept on high-dose propofol and fentanyl, but there were repeated cycles of sudden persistent severe hypercarbia, severely decreased pulmonary compliance and episodic breath holding. Eventually a suspicion of fentanyl-induced chest wall rigidity was made after excluding causes of airway resistance and reduction in pulmonary compliance. Gradual reduction in fentanyl infusion was associated with a reduction of episodes of reduced lung compliance and improvement in ventilation. Fentanyl is often used for analgesia and sedation in the ICU. It has a good side
\end{abstract}

Manuscript submitted July 29, 2019, accepted August 13, 2019

aDepartment of General Medicine and Intensive Care Medicine, Seng Kang General Hospital, 110 Sengkang E Way, Singapore

bDepartment of General Medicine, Khoo Teck Puat Hospital, 90 Yishun Central, Singapore

${ }^{\mathrm{c} C o r r e s p o n d i n g ~ A u t h o r: ~ P e h ~ W e e ~ M i n g, ~ D e p a r t m e n t ~ o f ~ G e n e r a l ~ M e d i c i n e ~ a n d ~}$ Intensive Care Medicine, Seng Kang General Hospital, 110 Sengkang E Way, Singapore 544886, Singapore. Email: peh.wee.ming@singhealth.com.sg

doi: https://doi.org/10.14740/jmc3351 effect profile but it is not without harm. High doses of fentanyl can lead to dramatic worsening of respiratory mechanics that may be life threatening. Fentanyl-induced chest wall rigidity is an important side effect that needs to be considered in the differential diagnosis of respiratory failure in the ICU.

Keywords: Fentanyl; Chest wall rigidity; Respiratory failure

\section{Introduction}

Chest wall rigidity secondary to fentanyl usage is a recognized side effect of fentanyl. It presents with sudden onset of respiratory failure, hypercarbia and worsening of lung compliance. It is a medical emergency but is difficult to diagnose as there are many differential diagnoses to this presentation. There have been case reports of chest wall rigidity reported as complications of post-operative fentanyl usage as analgesia. Complications have also occurred when it has been used also as a sedative and analgesic agent for short procedures like bronchoscopy.

Here we describe a case of a patient with a prolonged stay in the intensive care unit (ICU) with repeated sudden onset of type 2 respiratory failure while on high doses of sedation and analgesia with fentanyl and propofol. This was eventually attributed to fentanyl-induced chest wall rigidity.

\section{Case Report}

The patient is an 80-year-old woman, who presented to the hospital with fever and productive cough with yellowish expectoration of 2 days duration. She also had lethargy over the same period and had sick contacts in the form of two daughters who both had recently recovered from a "flu-like" illness. She was known to have bronchial asthma treated with seretide 25/250 two puffs ON + PO monteleukast $10 \mathrm{mg} \mathrm{ON}$, hypertension treated with PO losartan $50 \mathrm{mg}$ BD, type 2 diabetes mellitus controlled with PO linagliptin $5 \mathrm{mg}$ OM and a previous right thalamic ischemic stroke 5 years ago for which she was on PO clopidogrel $75 \mathrm{mg} \mathrm{OM}$ and PO simvastatin.

The patient had a total of two admissions to the medical ICU. The patient was initially admitted to general ward for treatment of possible pneumonia and asthma exacerbation and started empirically on intravenous amoxicillin/clavulanic acid 
but had worsening shortness of breath and hypoxia in the ward requiring intubation and transfer to medical ICU for invasive ventilatory support.

She was put on volume control mode ventilation with a tidal volume (TV) of $8 \mathrm{~mL} / \mathrm{kg}$ ideal body weight and started on light sedation with intravenous propofol $(30-50 \mathrm{mg} / \mathrm{h})$ and fentanyl (30 - $50 \mu \mathrm{g} / \mathrm{h})$ infusions post-intubation for comfort. She was given intravenous (IV) hydrocortisone $100 \mathrm{mg} \times \mathrm{q} 6 \mathrm{~h}$ and inhaled bronchodilators for her asthma. Antibiotics were escalated empirically to IV meropenem. On day 2 of ICU stay, propofol and fentanyl infusions were discontinued and she was switched to pressure support mode of ventilation. She was given a total of 10 days of carbapenem cover. She required a slow wean from the ventilator over the next few days and was extubated to non-invasive ventilation (NIV) on day 7 . Trials to wean her off NIV were unsuccessful.

The patient required re-intubation for decompensated type 2 respiratory failure after 2 weeks of weaning trials in the wards. She was put on pressure-regulated volume-controlled (PRVC) mode ventilation and started on low-dose fentanyl and propofol infusions for sedation and analgesia. Serial chest X-rays (CXRs) done were suggestive of hospital-acquired pneumonia and the patient was treated with empiric IV antibiotics (piperacillin + tazobactam) and inhaled bronchodilators (salbutamol/ipratropium) with IV steroids for possible asthma exacerbation.

On day 3 of her second ICU stay, the patient required increasing doses of sedation due to agitation. Sedation and analgesia were increased gradually from $50 \mathrm{mg} / \mathrm{h}$ of propofol to $100 \mathrm{mg} / \mathrm{h}$ of propofol. Fentanyl was also increased from 30 to $100 \mathrm{mg} / \mathrm{h}$. Paradoxically, on increasing her sedation and analgesia, the patient was noted to have episodic severe discordant breathing with prolonged expiratory phase with breath-holding with severe ventilator desynchrony. Peak airway pressures were high $\left(>50 \mathrm{~cm} \mathrm{H}_{2} \mathrm{O}\right)$ and generated TVs were low (about $50-100 \mathrm{~mL}$ ) despite increasing pressure limits on the ventilator. No wheezing was heard on auscultation of her chest and plateau airway pressure remained low $\left(15-20 \mathrm{~cm} \mathrm{H}_{2} \mathrm{O}\right)$. The endotracheal tube was patent and airway secretions were minimal. Repeat CXR done did not show any evidence of pneumothorax or new infiltrates. Electrocardiogram (ECG) and cardiac enzymes performed did not show any acute changes. Multiple ventilatory modes were tried (pressure control, volume control, PRVC) without any improvement in ventilation. Because of extremely low TVs, the ventilator was changed to exclude a leak which was less likely given the high airway pressures. She was empirically treated with IV steroids and also given multiple puffs of bronchodilator with no significant improvement. End-tidal carbon dioxide tracing showed rising trend and serial arterial blood gas showed worsening respiratory acidosis ( $\mathrm{pH} 7.27, \mathrm{pCO}_{2}$ 52.9). She was put on increasing doses of sedation (propofol $150 \mathrm{mg} / \mathrm{h}$ and fentanyl $160 \mu \mathrm{g} / \mathrm{h}$ ). She responded well to boluses of muscle relaxants with subsequent improvement in ventilation and lowering of airway pressures dramatically. A magnetic resonance imaging (MRI) of the brain was done and did not show any central cause for the hypercarbia.

From day 3 to 4 , an attempt was made to wean down the propofol infusion. However, all efforts at weaning off the propofol infusion were met by repeat episodes of ventilator desynchrony, low minute volumes and breath-holding. Each episode of high peak pressure and low minute volumes was evaluated systemically. There was no response to bronchodilators, no worsening of the CXR and no pneumothorax. These episodes were aborted with small boluses of propofol of 10 - $20 \mathrm{mg}$. At this time, a diagnosis of possible fentanyl-induced chest wall rigidity was considered and the patient was taken off fentanyl and continued on propofol infusion. IV dexmedetomidine infusion was started for the patient with the propofol infusion. A dramatic improvement in breathing pattern was noted. There were no more episodes of ventilator asynchrony, low TVs and high airway pressures. The propofol infusion was weaned down with the dexmedetomidine infusion. If there were episodes of agitation, they were briefly aborted with boluses of propofol. The patient was switched to pressure support mode ventilation the next day with no further episodes of desynchrony.

Because of prolonged ventilation and difficulty in weaning as a result of deconditioning for repeated ICU admission, the patient underwent an elective tracheostomy. She was given low-dose IV fentanyl peri-tracheostomy and tolerated it well without any further respiratory distress. Further ICU stay was uneventful and she was gradually weaned off the ventilator completely and transferred out of ICU.

\section{Discussion}

Chest wall rigidity secondary to opioid usage is an uncommon side effect of using fentanyl that was first described in 1953 [1]. Majority of case reports in opioid-induced chest wall rigidity were done describing patients who developed it during induction of anesthesia [2-9]. There have been episodes of chest wall rigidity that have been reported developed after patients were administered small doses of fentanyl pre-procedure for bronchoscopy [10].

In ICUs, fentanyl is commonly used as analgesia and also for sedation. The incidence of chest wall rigidity secondary to fentanyl is unknown and likely to be under-reported as this is a diagnosis of exclusion that is clinically determined. Majority of cases have been reported as a result of using large doses of fentanyl after surgery in the surgical ICU $[6,7]$. In our case, fentanyl-induced chest wall rigidity occurred during the usage of fentanyl for sedation and analgesia in routine ICU management.

Doctors have attempted to manage fentanyl-induced chest wall rigidity via two methods. One is the administration of opioid antagonist naloxone. The second method is the administration of neuromuscular blocking agents [7, 11, 12]. In these cases, the effect of the treatment is dramatic. The reversal of the pathophysiology is quick and ventilation normalization occurs quickly.

Opioid-induced chest wall rigidity is characterized by increase in muscle tone in the thoracic and abdominal muscles after exposure to fentanyl. This leads to difficulty in ventilation. Diagnosis is challenging as there is no objective measure of measuring muscle rigidity. In our patient, she was obese and 
the additional fat tissue makes assessment even more subjective. Hence a high degree of clinical suspicion is required for diagnosis.

This phenomenon can happen both with high doses and low doses of fentanyl. Fentanyl-induced chest wall rigidity has usually been reported with high doses of fentanyl $(17 \mu \mathrm{g} / \mathrm{kg})$ [13]. In our case, the patient was administered fentanyl up to $180 \mu \mathrm{g} / \mathrm{h}$ as an infusion for a prolonged duration, extending the half-life to 12 to $14 \mathrm{~h}$ because of context-sensitive half-life. Bolus was also given to the patient. In the patient's first admission, a low dose of fentanyl of $50 \mu \mathrm{g} / \mathrm{h}$ was administered to the patient without any issue. In her case, high-dose exposure to fentanyl triggered off the chest wall rigidity. But it is also known that chest wall rigidity can develop even with small boluses of $100 \mu \mathrm{g}$ of fentanyl [14].

We have reported a case of fentanyl-induced chest wall rigidity as a cause of acute type 2 respiratory failure in the ICU that was diagnosed late in the course of illness. This diagnosis is difficult as sudden onset of type 2 respiratory failure with minimal TVs but extremely high airway pressures has many confounding differential diagnoses. A systematic approach is required in the evaluation as the diagnosis of fentanyl-induced chest wall rigidity is a diagnosis of exclusion. The patient was evaluated for asthma exacerbation, bronchospasm, inapt ventilator settings, pneumothorax, worsening of lung compliance secondary to pneumonia and even an underlying central neurological disorder. There was no wheezing noted on clinical examination. The patient was still treated with bronchodilators. Issues with ventilator were evaluated by disconnection which did not demonstrate dynamic hyperinflation and excluded leakage. The endotracheal tube was checked and all kinks or obstruction to the airway tube were excluded. The ventilator was also changed. Lung ultrasound did not support the diagnosis of pneumothorax. This was confirmed with a CXR, which did not suggest worsening pneumonia or new consolidation.

None of the investigations yielded significant findings leading to a diagnosis. The diagnosis of chest wall rigidity was considered late and until the diagnosis was considered, the strategy to abort this sudden presentation of patient-ventilator asynchrony was the usage of neuromuscular blocking agents and small boluses of propofol. The patient continued to experience acute worsening of respiratory function which was not ideal from the management point of view. Improvement only occurred when fentanyl was discontinued completely and this was over a $24-\mathrm{h}$ period.

The near-continuous infusion of high doses of fentanyl that the patient received likely contributed to a prolonged effect of fentanyl in the body system because of a lengthened context-sensitive half-life. In her case, the fentanyl was replaced with the usage of infusion dexmedetomidine. In other cases of fentanyl-induced chest wall rigidity, physicians used morphine infusions and hydromorphone infusions in place of fentanyl with success. Based on our experience, dexmedetomidine can also be considered as a reasonable alternative.

At this point, the pathophysiology of chest wall rigidity secondary to fentanyl remains unclear and is supported only by experimental studies. Mechanism of action for the chest wall rigidity includes activation of central mu receptors, stimulation of central dopaminergic and nor-adrenergic pathway [15-17].

\section{Conclusions}

Fentanyl is a common analgesic used in the ICU. It has a reasonable side effect profile. Chest wall rigidity is an uncommon side effect that can be treated effectively with muscle relaxant and naloxone. Most importantly, fentanyl needs to be withdrawn upon diagnosis. The experience shared in this case report highlights the need to consider chest wall rigidity as an iatrogenic cause of respiratory failure in the ICU in unexplained acute causes of respiratory failure even if the exposure to fentanyl is low. This will allow early diagnosis, timely intervention and reduce the length of time spent in the ICU and on the ventilator.

\section{Acknowledgments}

All personnel who have helped in this publication have been acknowledged.

\section{Financial Disclosure}

There is no financial disclosure to make.

\section{Conflict of Interest}

There is no conflict of interest.

\section{Informed Consent}

Informed consent has been taken from the patient.

\section{Author Contributions}

PWM and DLS contributed to the data collection; DLS drafted the article; PWM did critical revision of the article; PWM and DLS gave final approval of the version to be published.

\section{References}

1. Hamilton WK, Cullen SC. Effect of levallorphan tartrate upon opiate induced respiratory depression. Anesthesiology. 1953;14(6):550-554

2. Abrams JT, Horrow JC, Bennett JA, Van Riper DF, Storella RJ. Upper airway closure: a primary source of difficult ventilation with sufentanil induction of anesthesia. Anesth Analg. 1996;83(3):629-632.

3. Ackerman WE, Phero JC, Theodore GT. Ineffective ventilation during conscious sedation due to chest wall rigidity after intravenous midazolam and fentanyl. Anesth 
Prog. 1990;37(1):46-48.

4. Bennett JA, Abrams JT, Van Riper DF, Horrow JC. Difficult or impossible ventilation after sufentanil-induced anesthesia is caused primarily by vocal cord closure. Anesthesiology. 1997;87(5):1070-1074.

5. Bowdle TA, Rooke GA. Postoperative myoclonus and rigidity after anesthesia with opioids. Anesth Analg. 1994;78(4):783-786.

6. Caspi J, Klausner JM, Safadi T, Amar R, Rozin RR, Merin G. Delayed respiratory depression following fentanyl anesthesia for cardiac surgery. Crit Care Med. 1988;16(3):238-240.

7. Comstock MK, Carter JG, Moyers JR, Stevens WC. Rigidity and hypercarbia associated with high dose fentanyl induction of anesthesia. Anesth Analg. 1981;60(5):362363.

8. Hill AB, Nahrwold ML, de Rosayro AM, Knight PR, Jones RM, Bolles RE. Prevention of rigidity during fentanyl - oxygen induction of anesthesia. Anesthesiology. 1981;55(4):452-454.

9. Jaffe TB, Ramsey FM. Attenuation of fentanyl-induced truncal rigidity. Anesthesiology. 1983;58(6):562-564.

10. Phua CK, Wee A, Lim A, Abisheganaden J, Verma A. Fentanyl-induced chest wall rigidity syndrome in a routine bronchoscopy. Respir Med Case Rep. 2017;20:205-
207.

11. Coruh B, Tonelli MR, Park DR. Fentanyl-induced chest wall rigidity. Chest. 2013;143(4):1145-1146.

12. Viscomi CM, Bailey PL. Opioid-induced rigidity after intravenous fentanyl. Obstet Gynecol. 1997;89(5 Pt 2):822824.

13. Scamman FL. Fentanyl-O2-N2O rigidity and pulmonary compliance. Anesth Analg. 1983;62(3):332-334.

14. Dimitriou V, Zogogiannis I, Liotiri D, Wambi F, Tawfeeq N, Koumi A, Geldhof G. Impossible mask ventilation after an unusually low dose fentanyl-induced muscle rigidity in a patient with essential tremor: a case report and review of the literature. Middle East J Anaesthesiol. 2014;22(6):619-622.

15. Vankova ME, Weinger MB, Chen DY, Bronson JB, Motis V, Koob GF. Role of central mu, delta-1, and kappa-1 opioid receptors in opioid-induced muscle rigidity in the rat. Anesthesiology. 1996;85(3):574-583.

16. Lui PW, Lee TY, Chan SH. Involvement of coerulospinal noradrenergic pathway in fentanyl-induced muscular rigidity in rats. Neurosci Lett. 1990;108(1-2):183-188.

17. Lui PW, Lee TY, Chan SH. Involvement of locus coeruleus and noradrenergic neurotransmission in fentanyl-induced muscular rigidity in the rat. Neurosci Lett. 1989;96(1):114-119. 